Supporting Information for

\title{
Solvent-Dependent Core-Modified Rubyrin Self-Assembly at
}

\section{Liquid/Solid Interfaces}

Chen Chen, ${ }^{a, b}$ Siqi Zhang, ${ }^{a, b}$ Bin Tu, ${ }^{b}$ Ting Meng, ${ }^{b, c}$ Jianqiao Li, ${ }^{a, b}$ Yuxin Qian, ${ }^{a, b}$ Pengfei Li, ${ }^{c}$ Bo Liu, ${ }^{a}$ Wubiao Duan, ${ }^{*} a$ Haijun Xu, ${ }^{*}, c$ Fengying Zhao, ${ }^{*}, e, f$ Yang Peng, e,f Jing Li, e,f and Qingdao Zeng ${ }^{*}, b, d$

a Department of Chemistry, School of Science, Beijing Jiaotong University, Beijing 100044, China. E-mail: wbduan@bjtu.edu.cn (W.D.)

${ }^{\mathrm{b}}$ CAS Key Laboratory of Standardization and Measurement for Nanotechnology, CAS Center for Excellence in Nanoscience, National Center for Nanoscience and Technology (NCNST), Beijing 100190, China. E-mail: zengqd@,nanoctr.cn (Q.Z.)

${ }^{c}$ College of Chemical Engineering Jiangsu Key Lab of Biomass-based Green Fuels and Chemicals Nanjing Forestry University Nanjing 210037, China. E-mail: xuhaijun@,njfu.edu.cn (H.X.)

${ }^{\mathrm{d}}$ Center of Materials Science and Optoelectonics Engineering, University of Chinese Academy of Sciences, Beijing 100049, China.

eJiangxi College of Applied Technology, Ganzhou 341000, China. E-mail: fengying2599@sina.com (F.Z.)

${ }^{\mathrm{f}}$ Engineering Research Center of Nano-Geo materials of Ministry of Education, China University of Geosciences, Wuhan 430074,China.

\section{STM Investigation and Theoretical Calculations.}

2. STM images of the supramolecular assembled structures of $\mathrm{N}_{2} \mathrm{~S}_{4}-\mathrm{OR}(1)$ and $\mathrm{N}_{2} \mathrm{Se}_{4}-\mathrm{OR}(2)$ at the HA/HOPG interface. 


\section{STM Investigation and Theoretical Calculations.}

STM Investigation. STM investigation was performed by a Nanoscope IIIa (Bruker, USA) in ambient environment on solid/liquid interfaces. 1-phenyloctane(PO) and 1-heptanoic $\operatorname{acid}(\mathrm{HA})$ were used as solvent in this work. Firstly, the core-modified rubyrin: $\mathrm{N}_{2} \mathrm{~S}_{4}-\mathrm{OR}(1)$, $\mathrm{N}_{2} \mathrm{Se}_{4}-\mathrm{OR}(2), \mathrm{N}_{2} \mathrm{~S}_{4}-\mathrm{OR}(3)$ and $\mathrm{N}_{2} \mathrm{Se}_{4}-\mathrm{OR}(4)$, were dissolved separately in PO or HA to the saturated concentration, respectively. The usual saturated concentration was about $10^{-3} \mathrm{M}$ then diluting the solutions into $10 \%$ saturation. (There was no found of influence from different concentrations through our work, so all concentrations were less than $10^{-4} \mathrm{M}$ to get better STM images.) A droplet $(\sim 0.5 \mu \mathrm{L})$ of the solution was then dripped onto a freshly-cleaved HOPG surface. The image was obtained at the liquid/solid interface after immersing the tip into the solution. Text presented STM images were recorded using mechanically cut Pt/Ir (80/20) tips under constant current mode. The STM images presented were raw data without further modification.

Theoretical Calculations. Density functional theory (DFT-D3) using DMol3code ${ }^{1}$ was utilized to do the theoretical calculations. The $2 \mathrm{D}$ periodic structure on the graphite was described by the periodic boundary conditions (PBC). In the local spin density approximation (LSDA), the local exchange correlation energy from the Perdew and Wang parameterization $^{2}$ and Perdew-Burke-Ernzerh parametrization ${ }^{3}$ were adopted to trace exchange and correlation. In a local atomic orbital basis, all-electron spin-unrestricted Kohn-Sham wave functions were expanded. The calculations were all-electron ones, which were all performed with the medium mesh. For the large system, numerical basis set was used. A convergence criterion of $10^{-5}$ a.u. on the energy and electron density was set for 
the self-consistent field procedure. The unit cell parameters and the geometry of the adsorbates in the unit cell were optimized according to the experimental results. Optimized parameters and the interaction energy between adsorbates could be obtained after the energy and density convergence criterion were reached.

The model system was designed to demonstrate the interactions between the adsorbates and substrate. In this work, the adsorption of adsorbates on graphite was considered as similarity to that of graphene so that we used PBCs to do calculations on infinite graphene monolayers. In the superlattice, graphene layers were separated by the normal direction of $40 \AA$. When modeling the adsorbates on grapheme, we used Graphene supercells and the Brillouin zone sampled by a $1 \times 1 \times 1 \mathrm{k}$-point mesh. The interaction energy ( $\left.\mathrm{E}_{\text {inter }}\right)$ of adsorbates on graphite was $E_{\text {inter }}=E_{\text {tot(adsorbates/graphene) }}-E_{\text {tot(isolated adsorbates in vacuum) }}-$ $\mathrm{E}_{\text {tot(graphene). }}$ 
2. STM images of the supramolecular assembled structures of $\mathrm{N}_{2} \mathrm{~S}_{4}-\mathrm{OR}(1)$ and $\mathrm{N}_{2} \mathrm{Se}_{4}-\mathrm{OR}(2)$ at the HA/HOPG interface.

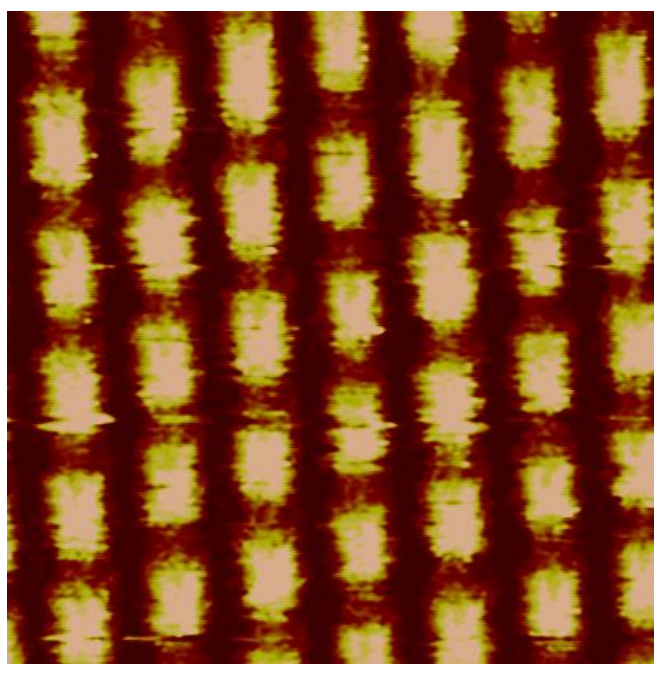

Figure S1. High-resolution STM image $\left(15 \times 15 \mathrm{~nm}^{2}\right)$ of $\mathrm{N}_{2} \mathrm{~S}_{4}-\mathrm{OR}(1)$ at the HA/HOPG interface. $I_{\text {set }}=150.8 \mathrm{pA}, V_{\text {bias }}=536.2 \mathrm{mV}$

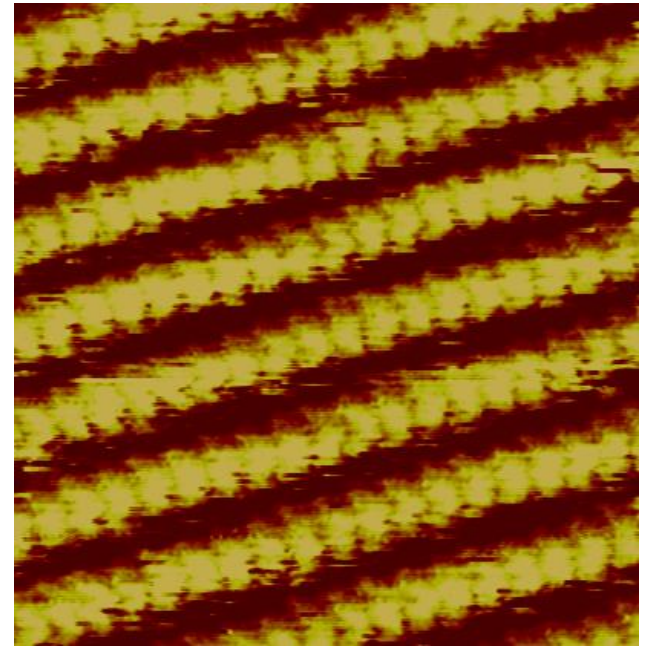

Figure S2. Large scale STM image $\left(35 \times 35 \mathrm{~nm}^{2}\right)$ of $\mathrm{N}_{2} \mathrm{Se}_{4}-\mathrm{OR}(2)$ at the HA/HOPG interface. $I_{\text {set }}=153.4 \mathrm{pA}, V_{\text {bias }}=560.2 \mathrm{mV}$. 


\section{REFERENCES}

1. Delley, B. From Molecules to Solids with the Dmol ${ }^{3}$ Approach. J. Chem. Phys. 2000, 113, 7756-7764.

2. Perdew, J. P.; Wang, Y. Accurate and Simple Analytic Representation of the Electron-Gas Correlation Energy. Phys. Rev. B. 1992, 45, 13244-13249.

3. Perdew,J. P.; Burke, K.; Ernzerhof, M. Generalized Gradient Approximation Made Simple. Phys. Rev. Lett. 1996, 77, 3865-3868. 